\title{
Lectoescritura como sistema neurocognitivo
}

\section{José María Gil}

https://orcid.org/00oo-0002-1216-7110

Universidad Nacional de Mar del Plata

Consejo Nacional de Investigaciones

Científicas y Técnicas (Conicet)

josemaria@gilmdq.com

jmgil@mdp.edu.ar

\section{Resumen}

Se realiza un análisis de la lectoescritura en general, de la conciencia fonológica, de los fundamentos de la neuroanatomía del lenguaje y de la dislexia. Gracias a ese análisis puede sugerirse que en torno al circuito del habla interna se configura otro circuito más amplio: el de la lectoescritura interna. A su vez, este circuito de la lectoescritura interna permite comprender que la conciencia fonológica involucra una serie de interconexiones entre cuatro sistemas: los de producción ortográfica y fonológica; $y$ los de reconocimiento fonológico y ortográfico (con particular énfasis en los dos últimos sistemas, para el caso de la lectura sola); más la caracterización del sistema de la lectoescritura y de la conciencia fonológica en términos neurocognitivos. Ello sirve para sugerir que los docentes deberian seguir trabajando con rimas, adivinanzas y canciones. La razón es que todas ellas contribuyen decisivamente a la estimulación y al desarrollo de la conciencia fonológica, la cual, por su parte, resulta fundamental para el aprendizaje de la lectura.

\section{Palabras clave (Fuente: tesauro de la Unesco)} Aprendizaje de la lectura; cognición; conciencia fonológica; didáctica del lenguaje; enseñanza de la lectura; fonética; proceso cognitivo.

Recepción: 11/07/2018 | Envío a pares: 24/04/2019 | Aceptación por pares: 01/10/2019 | Aprobación: 08/10/2019 


\title{
Literacy as a Neurocognitive System
}

\begin{abstract}
Literacy in general, phonological awareness, the foundations of language neuroanatomy and dyslexia are analyzed. Accordingly, it can be suggested that a wider circuit is configured around the internal speech circuit: the internal literacy circuit. This internal literacy circuit allows us to understand that phonological awareness involves a series of interconnections among four systems: orthographic and phonological production; phonological and orthographic recognition (with special emphasis on the last two systems, in the case of reading alone); the characterization of the literacy system and phonological awareness in neurocognitive terms. This indicates that teachers should continue working with rhymes, riddles and songs because they contribute decisively to stimulating and developing phonological awareness, which is in turn fundamental to learning to read.
\end{abstract}

\section{Keywords (Source: Unesco Thesaurus)}

Reading learning; cognition; phonologic awareness; language didactic; reading teaching; phonetics; cognitive process. 


\section{Lectoescrita como sistema neurocognitivo}

\section{Resumo}

Realiza-se uma análise da lectoescrita em geral, da consciência fonológica, dos fundamentos da neuroanatomia da linguagem e da dislexia. A partir dessa análise, sugere-se que, em torno do circuito da fala interna, seja configurado outro circuito mais amplo: o circuito da lectoescrita interna. Este permite compreender que a consciência fonológica envolve interconexões entre quatro sistemas: a produção ortográfica e fonológica; o reconhecimento fonológico e ortográfico (com ênfase nos últimos dois sistemas, para o caso da leitura sozinha); a caracterização do sistema da lectoescrita e a caracterização da consciência fonológica em termos neurocognitivos. Isso serve para sugerir que os docentes devam continuar trabalhando com rimas, adivinhações e cantigas, já que isso contribui decisivamente para estimular e desenvolver a consciência fonológica, a qual, por sua vez, é fundamental para a aprendizagem da leitura.

\section{Palavras-chave (Fonte: tesauro da Unesco)}

Ensino de leitura; cognição; consciência fonológica; ensino de línguas; aprendizagem da leitura; fonética; processo cognitivo. 


\section{Introducción}

Cuando un niño está aprendiendo a leer, llega a un cierto momento en el que puede identificar la representación gráfica de los sonidos del habla y empieza a entender que los símbolos alfabéticos se corresponden con los sonidos que se perciben o se emiten. Este descubrimiento maravilloso es el inicio de la conciencia fonológica, cuyo desarrollo y fortalecimiento serán esenciales para aprender a leer. En efecto, la enseñanza explícita de un sistema alfabético hace posible el desarrollo de la conciencia fonológica. De hecho, los adultos analfabetos manifiestan una clara tendencia a equivocarse en la detección de fonemas porque no tienen una conciencia fonológica desarrollada (Morais, Bertelson, Cary y Alegria, 1979; Morais, Cary, Alegria y Bertelson, 1986).

Es revelador también que, por lo general, los analfabetos tampoco entiendan juegos de palabras de base fonológica, ya sean intencionales o no buscados. Por ejemplo, no se dan cuenta de que el siguiente ejemplo es una broma o un equívoco: "Jesús es un pálido castor".

Entender que "Jesús es un pálido castor" (y no un "cálido pastor") es una broma o un equívoco requiere un buen grado de conciencia fonológica. De un modo comparable, los lectores adultos chinos que solo conocen el sistema de pictogramas también manifiestan una fuerte tendencia a fallar en las pruebas de conciencia fonológica. Por el contrario, los lectores chinos que además manejan el sistema de transcripción alfabética (llamado pinyin) obtienen buenos resultados en dichas pruebas (Mann, 1986; Read, Zhang, Nie y Ding, 1986; Cheung y Chen, 2004).

En este trabajo se intentará explicar por qué la conciencia fonológica y el aprendizaje de la lectura son procesos neurocognitivos. En primer lugar, ambos tienen su asiento en complejas y vastas redes que se despliegan en el cerebro (Lamb, 2011). De ahí el componente "neuro" para calificar a dichos procesos. Luego, el desarrollo de la conciencia fonológica y el aprendizaje de la lectura conforman sistemas -o, más bien, partes de sistemas o "sub-sistemas"- de conocimiento. De ahí su naturaleza "cognitiva".

Las investigaciones de las últimas décadas exhiben una copiosa evidencia a favor de la hipótesis de que la conciencia fonológica resulta muy favorable para aprender a leer. Dichas investigaciones no se limitan a reforzar una hipótesis ya consolidada en las ciencias del lenguaje, sino que además ofrecen información muy pertinente sobre variados aspectos del aprendizaje (y aun de la enseñanza) de la lectura. A modo de ejemplo, con especial referencia al caso de niños hispanohablantes, pueden mencionarse: la intervención pedagógica, por lo general temprana, tendiente a favorecer el aprendizaje o a prevenir dificultades comunes (Márquez y De la Osa Fuentes, 2003; Herrera y Defior, 2005; Gómez, Duarte, Merchán, Aguirre y Pineda, 2007; Trías, Cuadro y Costa, 2009; Bizama, Arancibia y Sáez, 2011; Bizama, Arancibia y Sáez, 2013); la localización de la conciencia fonológica (Bravo, 2002); las dislexias y los trastornos fonológicos asociados (Carrillo y Alegría, 2009; Cerverae Ygual, 2003; Coloma, Cárdenas y De Barbieri, 2005; Jiménez, 2012); la importancia decisiva de la conciencia fonológica en el proceso educativo o en entornos terapéuticos (Núñez y Santamarina, 2014; Vargas y Villamil, 2007; Sastre, Celis, Roa y Luengas, 2017); la relación entre la conciencia fonológica y el pensamiento lógico-matemático (Solsona, Navarro y Aguilar, 2005).

Debe destacarse también que las investigaciones vienen confirmando desde hace tiempo que las dificultades para aprender a leer se correlacionan con algún déficit en el procesamiento de la información fonológica. Aquí se tratará de mostrar además que las actividades tradicionales con adivinanzas, poemas y canciones siguen teniendo valor, porque contribuyen a estimular de forma decisiva el surgimiento de la conciencia fonológica en los niños prelectores. Ahora bien, esta última propuesta puede sonar poco original. En efecto, es algo más que sabido que los textos con ritmo y rima, con un fuerte predominio de la "función poética", son valiosos en 
el Kindergarten o en el primer grado de la escuela primaria. Esta sugerencia tal vez haga recordar a Pierre Menard, el personaje de Borges (1941) que decidió escribir el Quijote hacia 1934. Sin embargo, a pesar de la obviedad o el absurdo aparentes, el proyecto de Menard tenía sentido porque en definitiva permitía entender, entre otras cosas, que el significado de un texto no se reduce a las supuestas intenciones del autor, sino que más bien está en la mente del lector. Como sugiere Sydney Lamb (2004, p. 296), no existe eso que podamos llamar el significado de un texto, pues, más bien, los elementos del texto activan significados en las mentes de los lectores.

Así las cosas, los educadores comprometidos con los niños de niveles inicial y primario conocen bien la importancia de enseñar adivinanzas, poemas y canciones. Sin embargo, estamos en un contexto muy complejo e intenso. Aunque el uso de las nuevas tecnologías ofrece recursos promisorios en educación, también conlleva riesgos y efectos secundarios que a menudo se minimizan o se pasan por alto. En este sentido, y más allá de que aboga por la erradicación de los medios digitales en las aulas, Manfred Spitzer (2014) muestra que las nuevas tecnologías pueden ocasionar perjuicios. Por ejemplo, el tipiado en teclados puede perjudicar el aprendizaje de la lectoescritura y, a su vez, una lectoescritura deficiente perjudica las estrategias de aprendizaje. No es impertinente señalar aquí que las nuevas tecnologías, Internet en general y las redes sociales en particular, no solo han cambiado de forma drástica las pautas del juego en el mundo contemporáneo (a veces para bien, a veces para mal), sino que además el fenómeno de la adicción a las nuevas tecnologías se manifiesta en niños y adolescentes de los más diversos entornos geográficos y socioculturales (Li y Chung, 2006; Young, 2007; Wu y Cheng, 2007; Ferraro, Caci, D’Amico y Di Blasi, 2007; Johnson, 2009; Yubero, Larrañaga, Navarro y Elche, 2018).

En conclusión, sabemos que las nuevas tecnologías involucran cambios muy positivos como el acceso a la información y la conectividad, pero tam- bién promueven riesgos conocidos y efectos colaterales que contrastan marcadamente con los beneficios. En este contexto, en verdad complejo e intenso, no resulta tan obvio entonces venir a proponer que ciertas actividades tradicionales deberían seguir haciéndose simplemente porque favorecen mucho más que otras el aprendizaje de la lectoescritura y, a través de ella, el aprendizaje en general. Así, por ejemplo, James y Engelhardt (2012) muestran que la ejercitación continua de la letra manuscrita favorece grandemente el normal aprendizaje de la lectoescritura y por ello sugieren que la escritura a mano tiene que priorizarse muy por encima del uso de teclados en los primeros años de escolaridad.

A continuación, se pasará revista a aspectos fundamentales de la conciencia fonológica y de la lectura en tanto sistema neurocognitivo. Después, se considerarán algunos ejemplos pertinentes para el estímulo de la conciencia fonológica en niños pequeños. Por último se expondrán las conclusiones.

\section{Las rutas de la lectura}

La capacidad de leer tiene su asiento en el área occipito-temporal ventral izquierda en el cerebro de toda persona alfabetizada (Dehaene, 2009). Dado que tiene una base neurológica y forma parte de nuestro de nuestro conocimiento, el sistema de la lectura es un sistema neurocognitivo. Así, en términos neurocognitivos, aprender a leer consiste en establecer conexiones entre el área occipito-temporal ventral y las áreas del lenguaje ya establecidas, por ejemplo con el área de reconocimiento fonológico. La Tabla 1 da cuenta de las localizaciones corticales de algunos subsistemas del lenguaje, por ejemplo la lectura, y de algún otro subsistema conectado con el lenguaje, por ejemplo la visión.

Así, por caso, un accidente cerebro-vascular (ACV) que afecte de algún modo la zona occipitotemporal ventral izquierda causará una pérdida de la habilidad de la lectura, es decir, alexia. El área de la lectura se encarga de identificar las cadenas de le- 
tras y después distribuye esta información visual invariante a diferentes zonas del hemisferio izquierdo. La Figura 1 permite empezar a visualizar de modo general la ubicación de la corteza de la lectura como así también su relación con otros sistemas neurocognitivos del hemisferio izquierdo.

\section{Tabla 1: Localizaciones corticales aproximadas de algunos subsistemas neurocognitivos}

\begin{tabular}{|c|c|}
\hline $\begin{array}{c}\text { Subsistema } \\
\text { neurocognitivo }\end{array}$ & Área cortical aproximada \\
\hline $\begin{array}{l}\text { Percepción auditiva } \\
\text { primaria }\end{array}$ & $\begin{array}{l}\text { Corteza auditiva primaria (en } \\
\text { la circunvolución temporal } \\
\text { superior) }\end{array}$ \\
\hline $\begin{array}{l}\text { Reconocimiento } \\
\text { fonológico }\end{array}$ & Área de Wernicke \\
\hline Léxico & $\begin{array}{l}\text { Circunvolución angular } \\
\text { (lóbulo parietal) y otras } \\
\text { áreas adyacentes al Área de } \\
\text { Wernicke }\end{array}$ \\
\hline Producción gramatical & $\begin{array}{l}\text { Lóbulo frontal, cerca (y en } \\
\text { dentro) del área de Broca }\end{array}$ \\
\hline Producción fonológica & Área de Broca \\
\hline $\begin{array}{l}\text { Producción } \\
\text { articulatoria }\end{array}$ & $\begin{array}{l}\text { Regiones de la boca y de la } \\
\text { lengua de la franja motora } \\
\text { (circunvolución pre-central, } \\
\text { lóbulo frontal) }\end{array}$ \\
\hline $\begin{array}{l}\text { Categorías de objetos } \\
\text { concretos }\end{array}$ & $\begin{array}{l}\text { Varias localizaciones en los } \\
\text { lóbulos temporal y parietal }\end{array}$ \\
\hline Percepción táctil & $\begin{array}{l}\text { Corteza somato-sensorial: } \\
\text { lóbulo parietal }\end{array}$ \\
\hline Visión & Lóbulo occipital \\
\hline $\begin{array}{l}\text { Atención de arriba } \\
\text { hacia abajo y lectura } \\
\text { secuencial }\end{array}$ & Región parietal posterior \\
\hline Lectura & $\begin{array}{l}\text { Región occipito-temporal } \\
\text { ventral }\end{array}$ \\
\hline
\end{tabular}

Fuente: adaptada de Lamb (2011, p. 528).

Todos los sistemas de escritura oscilan entre la representación adecuada del sonido y la transmisión rápida de significado. Esta tensión se manifiesta en el cerebro lector: cuando leemos, se complementan senderos muy diversos que procesan la información lingüística. La Figura 2 muestra que para ir desde la palabra escrita (abajo a la derecha) a su pronuncia- ción (abajo a la izquierda), nuestro cerebro se apoya en diferentes rutas. Cuando una palabra es regular, la ruta fonológica convierte las letras en sonidos del habla de forma directa. Cuando una palabra es irregular, como por ejemplo zanahoria, intervienen las rutas léxica y conceptual, porque el lector necesita representarse el significado que evoca la palabra leída.

\section{Figura 1: Sistemas neurocognitivos fundamentales en la corteza del lenguaje}

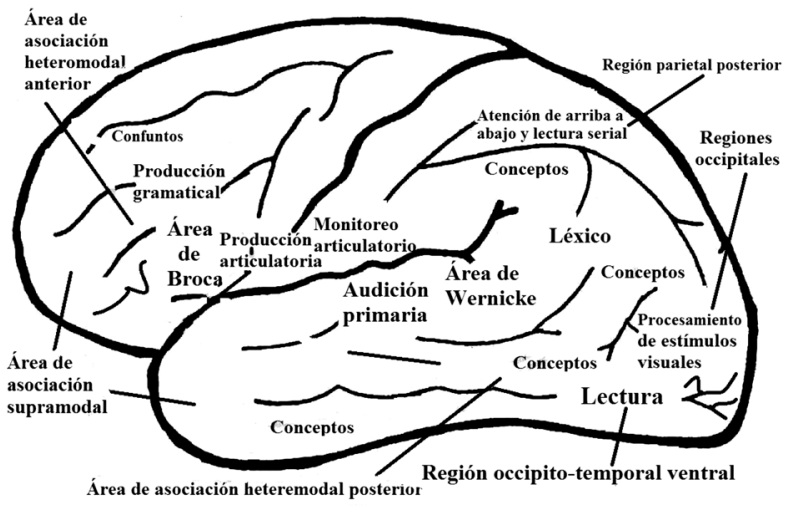

Fuente: adaptada de Lamb (2011, p. 527).

Figura 2: Las rutas de la lectura

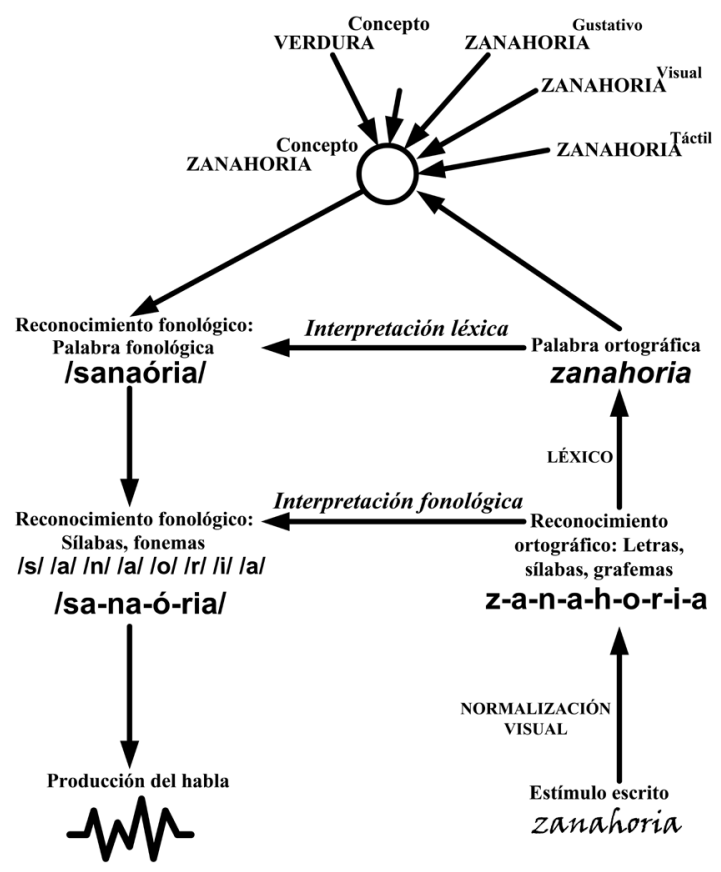

Fuente: reelaboración original basada en Dehaene (2009, p. 37). 
El marco teórico de esta propuesta es, como podrá advertirse, la teoría de redes relacionales desarrollada principalmente por el neurolingüista norteamericano Sydney Lamb y cuya sistematización final aparece en el libro Senderos del cerebro (2011). Investigaciones de avanzada en inteligencia artificial y teoría del conocimiento como las desarrolladas por John Sowa y colaboradores se basan en el supuesto de que la teoría de redes relacionales ofrece hipótesis contrastables con plausibilidad neurológica (Majumdar y Sowa, 2014; Sowa, 2016).

Por otra parte, la teoría de redes relacionales mantiene vigencia y vitalidad en debates sobre la naturaleza misma de la estructura del sistema lingüístico (Lamb 2016a/b). En el contexto de estos debates, Benzon (2016) entiende que no hay límites definidos entre las estructuras neurocognitivas del lenguaje y las estructuras del sistema cognitivo general, mientras que Osborne (2016) sostiene que un sistema de redes basado en la dependencia estructural sería más plausible en términos neurológicos. Hudson (2016) explica cómo las redes relacionales de Lamb son el antecedente de su propia "gramática de la palabra" y establece comparaciones en ese sentido. Por su parte, Rastall (2016) considera que las estructuras lingüísticas son producto de la tendencia natural del sistema cognitivo humano a sistematizar y clasificar.

Un análisis relacional y neurocognitivo arroja luz sobre la íntima vinculación que hay entre la conciencia fonológica y el sistema de la lectura, cuyo asiento está en el área occipito-temporal ventral del hemisferio izquierdo.

\section{Conciencia fonológica: lectoescritura, dislexia y fundamentos neurobiológicos}

La conciencia fonológica puede definirse como la representación mental de la capacidad de analizar y sintetizar los segmentos sonoros del habla. Se la reconoce como el mejor indicador y la mejor predicción del aprendizaje de la lectura. El desarrollo de la conciencia fonológica empieza bien temprano, ya en la edad preescolar, por ejemplo con el reconocimiento de rimas y con los juegos de palabras. En este sentido, varias investigaciones han mostrado desde hace tiempo que los niños del nivel preescolar que manifiestan fluidez en juegos fonológicos aprenden a leer más rápido (Rayner y Pollatsek, 1989; Lundberg, Frost y Petersen, 1988; Rayner, Foorman, Perfetti, Pesetsky y Seidenberg, 2001).

La conciencia fonológica permite manipular segmentos sonoros cada vez más finos hasta llegar al nivel del fonema. Las evaluaciones neurofisiológicas de la conciencia fonológica por lo general muestran claras diferencias en el funcionamiento de las áreas del lenguaje de personas disléxicas comparadas con lectores "normales". El desarrollo de la conciencia fonológica, entonces, facilita el aprendizaje de la lectoescritura y puede servir como medida preventiva de la dislexia, mientras que en los demás niveles educativos y en la educación de adultos la conciencia fonológica desempeña un papel importante en la rehabilitación (Mejía y Eslava, 2008). Ya en la primera mitad del siglo XX, Samuel Orton (1937) había advertido que los niños con problemas de lectura también tenían deficiencias en el habla y que estas deficiencias oscilaban entre sutiles y muy graves. Por ejemplo, algunos tartamudeaban o enfrentaban serias dificultades en el aprendizaje de lenguas extranjeras. Orton caracterizó estos errores como "latero-espaciales" y postuló que la causa debía ser una mala lateralización del lenguaje.

Después de la Segunda Guerra Mundial, las clasificaciones de dislexia dan cuenta de varios factores: problemas en la percepción del habla y en la discriminación de rasgos fonológicos (Johnson y Myklebust, 1967; Boder, 1973; Pirozzolo, 1979), dificultades en la articulación del habla y en la organización del acto motor que lasustenta (Mattis, French y Rapin, 1975), aspectos específicos de la comprensión o producción verbal (Azcoaga, Derman e Iglesias, 1997). En general, las clasificaciones de la dislexia incluyen subtipos ligados a la percepción del habla 
y algunos autores han afirmado que la dislexia involucra problemas de orden perceptivo (Kinsbourne y Warrington, 1962). También se ha debatido en profundidad la incidencia del desarrollo del lenguaje, en especial de la conciencia fonológica, en el aprendizaje de la lectoescritura (Goodman, 1982; Goodman y Goodman, 1979; Liberman, 1988).

Lo cierto es que las investigaciones efectuadas en diferentes contextos muestran que el desarrollo de la conciencia fonológica favorece el aprendizaje de la lectoescritura (Liberman, Shankweiler, Fischer y Carter, 1974; Blachman, 1989; Adams, 1990; Torgesen, Morgan y Davis, 1992; Catts, 1993). En este sentido, Goswami (1986) muestra que hay niños angloparlantes de cinco años que descubren de forma espontánea las analogías estructurales de ciertas palabras, por ejemplo pueden leer bean o peak porque antes han aprendido a leer beak. Así, las investigaciones ponen de manifiesto que las dificultades para el desarrollo de la conciencia fonológica permiten predecir dificultades en el aprendizaje de la lectura (y en gran medida a causa de ello) en el aprendizaje escolar (Catts, 1996). Por ejemplo, Bird, Bishop y Freeman (1995) observan, tras un seguimiento de 21 meses, que las mismas dificultades fonológicas que ciertos niños experimentan en la oralidad se manifiestan en el aprendizaje de la lectoescritura. Sugieren por ello que tanto el desorden de habla como la dificultad lectora tienen un factor común subyacente, que es la dificultad para analizar sílabas en unidades fonológicas menores.

Conviene señalar también que en el desarrollo de la conciencia fonológica interviene el condicionamiento cultural. Por ejemplo, Paul, Murray, Clancy y Andrews (1997) señalan que la persistencia de los problemas fonológicos en niños con desarrollo tardío del lenguaje se relaciona con el nivel sociocultural al que pertenecen. Así, los niños en condiciones de vulnerabilidad tienden a enfrentar más dificultades en el desarrollo de la conciencia fonológica y, con ello, en el aprendizaje de la lectoescritura. El hecho no es sorprendente, si se tiene en cuenta la correlación entre los grupos socioeconómicos desfavorecidos y el manejo de dialectos sociales no estandarizados. Esto no dice nada de la inteligencia de los niños en situación de vulnerabilidad ni del potencial expresivo de los dialectos no estandarizados. Sin embargo, sí hay una correlación entre los dialectos no estandarizados y las dificultades en el aprendizaje de los registros escolares, simplemente porque en el sistema escolar se usa de modo predominante el dialecto estándar (Gil, 2017).

Durante el desarrollo de la lectoescritura, después de la "etapa logográfica", el niño ingresa a la "etapa alfabética", también conocida como etapa fonológica (Frith, 1985; Dehaene, 2009). Una vez en esta etapa, el niño aprende a reconocer que las palabras se descomponen en letras y que las letras se corresponden con los sonidos del habla. La conversión de grafema en fonema es el procedimiento fundamental de la etapa alfabética/fonológica: el niño ya les presta atención a los constituyentes más pequeños, como las letras, las sílabas y los arranques silábicos. De manera especial, conecta los grafemas con los sonidos del habla, lo cual le permite de algún modo jugar con el armado de palabras y aun deletrear palabras desconocidas. Se da entonces una profunda transformación cerebral cuando el niño descubre que la secuencia del habla se puede dividir en fragmentos menores, por ejemplo que el sonido [ma] consta de dos instancias sonoras [m] y [a], que en nivel de abstracción más alto cuentan como realizaciones de los fonemas $/ \mathrm{m} / \mathrm{y} / \mathrm{a} /$. Obviamente, el niño no necesita ni es capaz de verbalizar ese descubrimiento.

Así los primeros años de escolaridad lectora llevan al surgimiento de una representación explícita de los sonidos del habla. La instancia clave consiste en el descubrimiento de que el habla está formada por unidades básicas que pueden combinarse para crear palabras nuevas. Esta capacidad es, digámoslo una vez más, la "conciencia fonológica". Ahora bien, la conciencia fonológica resulta fundamental para aprender a leer. Y, por contrapartida, para tener conciencia fonológica se requiere de la enseñanza explícita de 
un alfabeto. Como se ha dicho, los adultos analfabetos suelen fallar en la detección de fonemas (Morais et al., 1979; Morais et al., 1986). Todo lo que el niño empieza a hacer en la etapa fonológica resulta posible gracias a cambios neurofisiológicos que tienen su asiento en la corteza cerebral. En efecto, cuando el niño empieza a distinguir una secuencia alfabética, las áreas visuales del cerebro aprenden a dividir la palabra en grafemas. Esto significa que algunas de las áreas del habla deben adaptarse a la representación explícita de fonemas y que dichas adaptaciones tienen que estar altamente coordinadas para que surja una ruta eficiente que convierta la letra en sonido.

Ya se han mencionado investigaciones que muestran que el dominio de -y aun el juego conlos sonidos del habla a una edad temprana mejora sensiblemente la conciencia fonológica y, con ello, el rendimiento en la lectura. Estos hallazgos han llevado a aceptar la hipótesis de que la conciencia fonológica es un requisito para el aprendizaje de la lectura. Dicho de un modo no poco interesante: el reconocimiento consciente de los fonemas precede al aprendizaje de los grafemas.

Debe admitirse, con todo, que hay cuestionamientos atendibles. Así, Castles y Colheart (2004), por ejemplo, creen que la relación causal entre la conciencia fonológica y el aprendizaje de la lectura no está definitivamente confirmada. Algunas investigaciones se llevaron a cabo con niños que ya conocían algunas letras, lo cual puede haberles facilitado la segmentación del habla. Castles y Colheart también destacan la influencia que ejerce el deletreo en el procesamiento fonológico. En efecto, muchos niños angloparlantes dicen que perciben tres sonidos en rich [rico, adinerado] y cuatro sonidos en pitch [tono o cancha], cuando de hecho las dos palabras constan de cuatro fonemas: Se transcriben /rit j/ y / prtf/, respectivamente. Según esta crítica, la conciencia ortográfica es lo que termina incidiendo en el reconocimiento fonológico. De un modo análogo, Ehri y Wilce (1980) sugieren que el conocimiento de la ortografía influye decisivamente en la percepción del habla.
De todas maneras, que el conocimiento de la escritura incida en el reconocimiento fonológico no constituye evidencia en contra de la importancia de la conciencia fonológica para el aprendizaje de la lectoescritura. Es del todo esperable que las conexiones establecidas en el área occipito-temporal ventral (el área de la lectura) empiecen a participar del reconocimiento fonológico, especialmente en el caso de los lectores ya formados o en el caso de los lectores expertos. En algún momento, el área de la lectura empieza a monitorear el reconocimiento fonológico de un modo comparable a aquel en que el reconocimiento fonológico ya monitoreaba el área de la lectura durante los inicios del aprendizaje. Una vez establecidas, las conexiones entre estas dos áreas se van fortaleciendo a lo largo de toda la vida de la persona lectora.

Por su parte, la dislexia consiste en los trastornos de lectura que padece un individuo, aun a pesar de que sus capacidades cognitivas son, como mínimo, normales. Desde hace bastante, las investigaciones vienen mostrando la correlación entre los varios tipos de dislexia y la conciencia fonológica. Así, Tallal (1980) y Tallal, Stark y Mellits (1985) sugieren que los problemas en el aprendizaje de la lectoescritura se deben a un déficit en el procesamiento temporal de la información sensorial auditiva. Dicho déficit altera de modo crítico el desarrollo de la conciencia fonológica y acarrea después numerosos problemas de aprendizaje. Algunos estudios muestran que la posible aparición de trastornos podría evaluarse en el primer año de vida y que el entrenamiento sistemático reduciría su impacto en el aprendizaje del lenguaje y de la lectoescritura (Merzenich et al., 1996; Tallal et al., 1996; Benasich y Tallal, 2002).

La concepción optimista de las pruebas y los entrenamientos con un fin preventivo deben igualmente tomarse con prudencia, si se considera que los problemas de conciencia fonológica están causados por defectos en los centros especializados del lenguaje. En este sentido, también se ha analizado 
con mucho recaudo la correlación entre dichos inconvenientes de base neurológica y los trastornos específicos de aprendizaje de lectura (Betherton y Holmes, 2003; Galaburda y Cestnick, 2003; Kaminsky, Eviatar y Norman, 2002; Waber et al., 2002; De Martino, Espesser, Rey y Habib, 2001).

Las comparaciones neurofisiológicas entre lectores "normales" y los lectores disléxicos muestran diferencias de activación de las zonas del lenguaje. Por ejemplo, en los disléxicos se observa una "sobreactivación" de las regiones anteriores y una activación disminuida en las regiones posteriores, todo lo cual sugiere una desconexión funcional en el sistema que sustenta el análisis fonológico que resulta crítico para la lectura (Shaywitz, Shaywitz, Pugh y Fullbright, 1998). Otros estudios con lectura de palabras simples y pseudopalabras muestran que la transformación explícita del grafema en fonema requiere la conexión entre la circunvolución angular izquierda y otras regiones, como las áreas visuales de asociación, la circunvolución temporal superior media y el área de Broca y cercanías, lo que no ocurre en adultos disléxicos (Horwitz, Rumsey y Donohue, 1998).

Para los déficits de procesamiento temporal de sonidos, Galaburda y Cetsnick (2003) reportan cambios en la composición de neuronas de núcleos geniculados laterales y mediales. Otros hallazgos establecen una relación significativa entre las dislexias con ciertas alteraciones de la conciencia fonológica como expresión fenotípica y anomalías del cromosoma 6 (Grigorenko et al., 1997).

Si las causas de la dislexia son de orden genético, el tratamiento de las personas que la padecen es evidentemente muy complicado, porque no puede haber, en principio, un conjunto de actividades o de estrategias didácticas que permitan corregirla. De todas formas, y aunque la causa de la dislexia fuera genética, la estimulación temprana de la conciencia fonológica sigue siendo útil para prevenir o superar los trastornos de la lectura.

\section{Desarrollo y estimulación de la conciencia fonológica: adivinanzas, rimas, canciones}

El reconocimiento de rimas, la identificación de los comienzos y los finales de las palabras, la separación en sílabas, la interpretación de adivinanzas y canciones, son todas actividades concretas y observables que los niños son capaces de hacer gracias al sistema lingüístico interno que tiene su asiento en el cerebro. De manera más específica, para que el niño pueda desplegar todas esas actividades, en el sistema lingüístico interno tiene que estar desarrollándose la conciencia fonológica. Así, hacia los tres años, los niños son en general capaces de reconocer dos palabras que riman, mientras que otras habilidades más complejas, como eliminar sonidos dentro de las palabras para descubrir una palabra nueva, se desarrollan recién hacia los 8 años (Yopp, 1988; Treiman y Zukowski, 1991). En este sentido, el modelo de Paul et al. (1997) reconoce las siguientes etapas en el desarrollo de la conciencia fonológica:

1. Identificación de rimas.

2. Apareamiento de sílabas.

3. Apareamiento de palabras por arranque silábico.

4. Segmentación de sonidos dentro de la palabra.

5. Manipulación del orden de segmentos dentro de las palabras.

6. Eliminación de sonidos dentro de la palabra.

Cada una de estas instancias incluye a su vez una secuencia que depende de la complejidad de las palabras y los segmentos a los cuales se enfrente el niño. Por ejemplo, es más fácil aparear por sonido inicial baño y barro que baño y burro.

La evaluación y el entrenamiento de la conciencia fonológica son fundamentales no solo para los docentes de los niveles iniciales, sino también para psicólogos, psicopedagogos, médicos y todos aquellos profesionales que se dediquen a la enseñanza o al cuidado sanitario de los niños preescola- 
res o del primer ciclo de la escuela primaria. Así, la evaluación de la conciencia fonológica contempla rimas, aliteraciones, pseudopalabras y otros aspectos comolos enumerados en las instancias 1- 6 de Paul (Reigosa, Pérez, Piñeiro y López, 2002). Por ejemplo, Fazio (1997a/b) llevó a cabo estudios de niños que padecían retardos del lenguaje para evaluar el aprendizaje de un poema con y sin el apoyo del movimiento corporal. Su conclusión fue que para todos los niños el apoyo gestual y motriz favorece el aprendizaje. Ahora bien, mientras que el grupo de control aprende el poema aun sin los movimientos de ayuda, para el grupo con retardo del lenguaje el apoyo del movimiento corporal cuenta como una diferencia favorable muy significativa.

Debe tenerse en cuenta que en el desarrollo de la conciencia fonológica intervienen una compleja variedad de procesos neurocognitivos. La lista que sigue se inspira en la que proponen Mejía y Eslava (2008, p. 61).

1. El reconocimiento fonológico permite distinguir los rasgos de los fonemas. Se localiza en el área temporal superior secundaria. (Se trata de un área secundaria porque asocia y procesa información de un único dominio cognitivo, en este caso, del sistema auditivo.)

2. El factor cinestésico distingue las posturas y los movimientos de los órganos articulatorios. Se localiza en el área secundaria parietal inferior (La zona es unimodal porque asocia información del sistema somato-sensorial.) Se procesan aquí sensaciones de origen muscular o articulatorio que brindan información sobre la posición de las diferentes partes del propio cuerpo en el espacio.

3. El factor cinético permite el paso fluido de un "articulema" a otro para dar como resultado una producción con melodía cinética y se ubica en las zonas frontales posteriores, en el área premotora. (Un articulema es la representación articulatoria de un fonema.)
4. La retención audioverbal mantiene activa la información fonológica a medida que se va procesando una secuencia. Se localiza en la zona temporal media.

5. El factor sucesivo posibilita la percepción de la secuencia fonológica. Se localiza en el área temporal superior secundaria y envía activación a otras zonas del lenguaje en la corteza, empezando por las áreas del léxico (cfr. Figura 1 y Tabla 1).

6. El factor simultáneo efectúa una sintesis de la secuencia fonológica que se percibe. Mejía Eslava y Eslava Cobos (2008) hacen mención del hemisferio derecho, donde se procesan aspectos como la entonación.

7. La regulación y el control permiten actuar con intencionalidad y conciencia. Se ubican en las zonas frontales terciarias o prefrontales, es decir, en las zonas heteromodales de asociación, donde ya se procesa información proveniente de diferentes sistemas cognitivos.

8. El factor neurodinámico estabiliza la producción del habla. Se localiza en zonas subcorticales profundas

Como en todo sistema funcional, cuando falla alguno de los factores, el sistema de conciencia fonológica se puede reorganizar sobre la base de los factores indemnes. Así se explican, por ejemplo, el desarrollo de la conciencia fonológica en sordos ( $\mathrm{Mi}$ ller, 1997; Leybaert, 1998; Sterne y Goswami, 2000) y las relaciones entre la conciencia fonológica y el aprendizaje lector por parte de estudiantes sordos (Nielsen y Luetke-Stahlman, 2002).

En este contexto, las adivinanzas constituyen un estimulante ejercicio para el surgimiento, el desarrollo o aun el fortalecimiento de la conciencia fonológica en niños pequeños. Consideremos algunos ejemplos: 
(1)

A mí me tratan de santa y mi nombre lleva el día, soy redonda y colorada, dicen que soy rica fría.

(2)

Se pasea por los mares, y es una grandota buena, como siempre come mucho, le decimos que va llena.

(3)

Una hoz parezco a veces, y a veces parezco un queso, tengo mar, no tengo peces, y no podés darme un beso.

(4) Juego con mis cuatro cuerdas, y mi arco bailarín. ¡Escuchá, no te lo pierdas! ¡Mi alegría no tiene fin!

(5)

Por medio de la Figura 3 se busca ilustrar cómo la adivinanza del texto (5) promueve un juego con la información conceptual, léxica y fonológica. En esta figura se utilizan convenciones de la notación fina de la teoría de redes relacionales (Lamb, 2011). Las flechas apuntan hacia arriba, porque se representa la activación en sentido ascendente (de la fonología al significado) para así dar cuenta del proceso general de reconocimiento. (Si se quisiera dar cuenta del proceso general de producción habría que diseñar otra figura, con las flechas en sentido descendente, es decir, desde el significado hacia la fonología.)

De manera general, la Figura 3 permite entender que en el sistema lingüístico de un niño que comprende la adivinanza se despliegan los siguientes procesos:

1. El sistema de reconocimiento fonológico (en el área de Wernicke, cfr. Tabla 1 y Figura 1) interpreta la secuencia correspondiente a "espera". Cada uno de los círculos negros en la parte inferior representa el punto de salida de activación de los nodos fonológicos que se realiza cuando el niño reconoce precisamente la secuencia "espera".

\section{Figura 3: Activación ascendente de nodos fonológicos, léxicos y conceptuales en la adivinanza de la PERA. Se representa el juego de palabras con "pera" y "espera"}

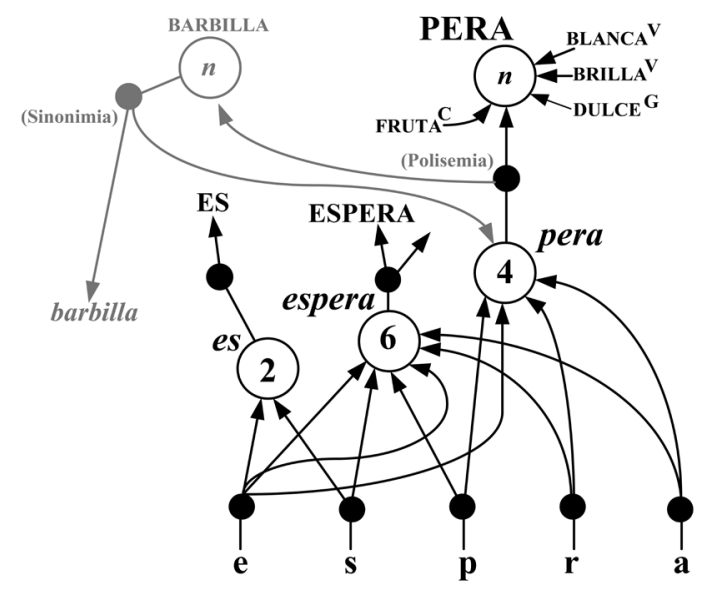

Fuente: elaboración propia.

2. La activación que sale de los nodos fonológicos (marcada por las flechas ascendentes) se dirige hacia las entradas del nodo léxico para espera y también hacia las entradas de los nodos para léxicos es y pera. Dichas entradas se representan por medio de círculos con números enteros en su interior. Los números indican el valor del umbral necesario para la activación: El valor $n$ especifica cuántas líneas de activación deben ingresar al nodo para que en efecto se active. Por ejemplo, el nodo léxico para pera tiene valor de umbral 4, porque se activa si y solo si llega la activación proveniente de los nodos para /p/, /e/, /r/y /a/ (que son 4).

3. La salida del nodo léxico para pera (representada con un círculo negro) envía activación para los conceptos PERA/FRUTA y PERA/BARBILLA simultáneamente. Es esperable que PERA se active con mucha más fuerza porque la adivinanza 5 promueve la activación de conceptos relativos al color, al brillo y al gusto. Obsérvese que, por ejemplo, el nodo conceptual para PERA tiene un umbral en cuyo interior figura $n$ y no un nú- 
mero entero. Por medio de esta notación se intenta mostrar que nunca puede definirse el número preciso de activaciones entrantes para un nodo conceptual. Por su parte, la salida del nodo léxico para espera activa el concepto de ESPERAR, que también es pertinente en este caso, porque la adivinanza juega con la ambigüedad de forma deliberada.

4. El nodo conceptual para BARBILLA y todas sus activaciones ascendentes y descendentes se representan en gris, porque en el sistema lingüístico del niño que interpretó la adivinanza han recibido una activación mucho más débil que el nodo conceptual para Pera. Así, la Figura 1 también sirve para mostrar que las redes relacionales dan cuenta de la estructura y la función lingüística. Por ejemplo, la sinonimia se explica como la conexión de un nodo conceptual con dos (o más) nodos léxicos; en este caso, el concepto BARBILLA se conecta en sentido descendente con los nodos léxicos para pera y barbilla. Por su parte, la polisemia se concibe como la conexión entre un nodo léxico y más de un nodo conceptual, por ejemplo la que se entre el nodo léxico para pera y los nodos conceptuales para PERA/FRUTA Y PERA/BARBILLA.

En síntesis, la interpretación de una adivinanza (como la de cualquier otro texto) involucra la activación de nodos fonológicos, léxicos y conceptuales. Lo que resulta de especial interés aquí, tal como se representa en la Figura 3, es que la secuencia fonológica "espera" activa senderos alternativos de manera simultánea, razón por la cual terminan activándose los conceptos de pera y esPERAR. En otras palabras, la activación de la fonología es lo que propicia y refuerza asociaciones pertinentes a nivel conceptual, vía el léxico. En este sentido, las adivinanzas constituyen en efecto un muy buen medio para empezar a desarrollar o fortalecer la conciencia fonológica.

Otro ejemplo valioso es el de las canciones. Podemos, por caso, considerar "El último tranvía", de María Elena Walsh.

\section{El último tranvía}

El último tranvía

que rueda todavía

se va, se va, se va.

Qué lástima me da,

pues ya no volverá.

Por un caminito de aserrín va el tranvía, tin tilín tilín.

Pide una manzana y no le dan

ni una esquina, tan talán talán.

Si un tranvía va por un jardín se equivoca, tin tilín tilín.

Y si choca con un capitán paga multa, tan talán talán.

Si a un tranvía le brota un jazmín en el techo, tin tilín tilín, las hormigas cómo viajarán de contentas, tan talán talán.

Si un tranvía toma naranjín se emborracha, tin tilín tilín.

Pero si un tranvía come pan no se empacha, tan talán talán'.

De manera informal, la Figura 4, a continuación, ilustra las conexiones bidireccionales entre los nodos léxicos que manifiestan rima y los conceptos tranvía y todavía. (Las líneas simplemente indican que hay una conexión de ida y vuelta entre los nodos involucrados.)

Resulta claro que la activación de los nodos fonológicos es fundamental para una interpretación. En efecto, las onomatopeyas "tin tilín tilín" y "tan talán talán" permiten sostener las imágenes del

$1 \quad$ Una versión en línea: María Elena Walsh. "Canción del último tranvía". En 20 Grandes Éxitos, vol. 1, 2000. YouTube. https://www.youtube.com/watch?v=JsfsluOjdIY\&t=74s 
tranvía. Al igual que las adivinanzas, las canciones pueden favorecer el surgimiento, el desarrollo o el robustecimiento de la conciencia fonológica, la cual, a su vez, favorece la comprensión.

Figura 4: Conexiones entre nodos léxicos y conceptuales en "El último tranvía"

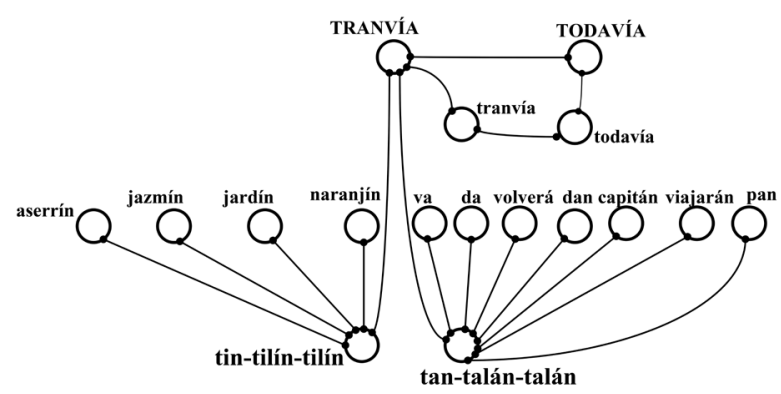

Fuente: elaboración propia.

\section{Conclusiones y propuesta: el circuito de la lectoescritura, la conciencia fonológica y la enseñanza en el nivel inicial}

La percepción del habla, que desde luego precede a la conciencia fonológica, es un proceso neurocognitivo. El sistema que la sustenta se ubica en el lóbulo temporal y se trata del área de Wernicke. Por su parte, la producción fonológica se localiza en el área de Broca. Aunque esto es harto sabido, no siempre se toma en cuenta que hay dos sistemas fonológicos, es decir, el sistema de reconocimiento fonológico y el sistema de producción fonológica.

Gracias a la evidencia neurológica se sabe también que la percepción es bidireccional, porque no va exclusivamente "de abajo hacia arriba". Por ejemplo, el sistema conceptual incide fuertemente en la percepción: si vemos la cabeza del gato asomándose por la ventana, interpretamos que está el gato entero, y no solo su cabeza. El sustrato anatómico de la percepción está dado por las conexiones bidireccionales (que se alimentan de forma retroactiva y prospectiva). Estas conexiones bidireccionales se desarrollan no solo "hacia arriba", es decir, desde los órganos sensoriales, sino también "hacia abajo", esto es, desde los patrones conceptuales. Por caso, si se me pide que visualice un tigre o una catarata, pue- do hacerlo porque el input lingüístico fue hasta mi sistema conceptual y así, por medio de un procesamiento descendente, activo el mismo subconjunto de conexiones neuronales que se hubieran activado en el caso de que hubiera visto in situ un tigre o una catarata. Lo mismo puede pasar si te pido, lector, que oigas (o recuerdes) el inicio de la Quinta Sinfonía de Beethoven, en el caso de que ya la conozcas.

Estos procesos descendentes también se dan sin que haya un input real. Pueden en efecto trabajar como respaldo (o distorsión) de la percepción ordinaria que es promovida por el input sensorial. Por ejemplo, si veo a una persona sentada tras un escritorio no concluyo lo siguiente: “¡Uy, ahí están la cabeza y el torso de Lorena! Me pregunto si estarán cerca su cintura y sus piernas". No pensamos ni decimos eso porque la imagen perceptiva se completa con nuestro conocimiento, gracias a las conexiones en nuestro sistema conceptual, el cual permite que la activación se esparza hacia abajo a través de nuestros sistemas perceptivos.

En un sentido comparable, la percepción visual se despliega de manera específica en la corteza visual (en el lóbulo occipital), pero en realidad otras áreas participan de la percepción visual, las motoras incluso. De especial interés para los docentes de nivel inicial es que la percepción auditiva no se desarrolla únicamente en la corteza auditiva. Como toda parte de la corteza, se relaciona, aunque sea de forma mediada, con toda otra parte de la corteza, y como las diferentes partes son capaces de trabajar de forma paralela, lo cierto es que la percepción del habla se despliega en una variedad de subsistemas corticales.

En efecto, las estructuras corticales involucradas en la percepción del habla son la visual, la motora y la conceptual. Tal como sugiere el neurolingüista norteamericano Sydney M. Lamb (2004, p. 355), a muchos puede sonarles sorprendente que las estructuras motoras estén involucradas en la percepción o que las habilidades perceptivas participen en la actividad motora. Pero, si se analiza el tema 
con cuidado, nos damos cuenta de que, por ejemplo, para escribir la letra "A" (un proceso regulado por la corteza motora) no solo tengo que tener la representación perceptiva de la letra "A", sino que, a menos que escriba con los ojos cerrados, también debo monitorear mi propia escritura de la letra, todo lo cual está regulado por la corteza perceptiva, en el área de la lectura, que tiene su asiento en el área occipitotemporal ventral.

Deberíamos distinguir entonces la micropercepción de la macropercepción. Así, la micropercepción de la palabra escrita tiene lugar en la corteza occipito-temporal ventral, como ya hemos visto. Por su parte, la macropercepción en la lectura es el proceso global de reconocimiento de la palabra escrita en el que participan activamente otras áreas de la corteza. De modo análogo, podemos distinguir microproducción, o procesamiento micromotor, de macroproducción o macromotor.

Sin embargo, algunos investigadores han cuestionado por ejemplo que el reconocimiento fonológico tenga su asiento en un área específica, tal como es el área de Wernicke. Por ejemplo, Steven Pinker escribe lo siguiente: "se pensó alguna vez que en el área de Wernicke subyacía la comprensión del lenguaje. Pero eso no podría explicar por qué el habla de esos pacientes afásicos suena tan psicótica" (1994, p. 311). En otras palabras, la pregunta de Pinker sería esta: ¿cómo puede ser que, si lo que está dañado es el sistema de reconocimiento y no el sistema de producción, justamente la producción verbal de los afásicos de Wernicke sea tan defectuosa? Considérese por ejemplo la siguiente transcripción de las investigadoras Beatriz Gallardo y Julia Sanmartín del pasaje defectuoso de un hablante que padecía una afasia sensitiva posterior a un tumor irradiado.

tenemos todos los elementos/ que nos funcionan// desde el agual la corriente eléctrical campos magnéticos// eeh todos los fenómenos esos sicológico/de la bondaad/de la voluntaad/ de esto y de los otro// todo está ahíi /// y si hay cAARga/ por-por un sitio y está corriendo/ hay campos magnéticos// y si hay campos magnéticos $\uparrow$ pues tienen una forma de actuar sobresobre-sobre las cosas/ que absorbe/ resolva/y a lo mejor se le podía dar/ por ahí/ cuestiones a los fenómenos/como la bondad/ el malestar/ las cosas. (2005, p. 188)

Ahora bien, que el habla de un afásico de Wernicke sea defectuosa no refuta la hipótesis de que el reconocimiento fonológico se localiza en el área de Wernicke, simplemente porque el reconocimiento fonológico es necesario para monitorear la producción del habla. Dicho de otra forma, necesitamos una comprensión intacta para monitorear nuestra producción. Por lo tanto, el área de Wernicke sí desempeña un papel importante en la producción. Para entender la función que desempeña el reconocimiento en la producción, que trate alguien de escribir algo sin mirar el papel y que compare luego lo que escribió en ese caso con su escritura habitual, cuando puede ir leyendo lo que escribe. De manera análoga, no hay que sorprenderse de que una persona con el reconocimiento fonológico dañado produzca un habla defectuosa.

Es así que, cuando habla, una persona dispone de dos vías para el monitoreo auditivo. Por un lado, oye las ondas sonoras que ella misma emitió y circulan por el aire; esta vía comienza en la producción y llega hasta los oídos y, luego, al reconocimiento fonológico. Por otro lado, esa misma persona monitorea internamente su propio discurso por medio del circuito del habla interna. Para ello se usan conexiones internas directas que van desde la producción articulatoria hasta la percepción auditiva y posiblemente se usan también conexiones que van de la producción fonológica al reconocimiento fonológico.

Ahora bien, de manera complementaria, la producción participa en los procesos perceptivos. Por ejemplo, cuando a una persona se le muestra la foto de una mano y se le pregunta si es la derecha o la 
izquierda, las neuroimágenes muestran que no solo hay actividad en la corteza visual, sino también en la corteza motora, precisamente en las partes que rigen los movimientos de las manos. Lo que ocurre es que cuando respondemos si la mano de una foto es la derecha o la izquierda nos imaginamos que ponemos una de las manos en la posición de la imagen. Este principio permite interpretar la célebre (y también discutida) teoría de las "neuronas en espejo" de Rizzolatti (Hickock, 2014; Rizzolatti y Sinigaglia, 2015).

En conclusión:

1. El sistema de reconocimiento fonológico (que es un sistema perceptivo) controla y monitorea la producción fonológica. En términos de Lamb (2011), la "micropercepción" del habla en el área de Wernicke desempeña un rol fundamental en la "macroproducción" del habla, porque la monitorea.

2. La corteza motora desempeña un rol fundamental en la percepción, ya que, al percibir su propio discurso, una persona hace uso del circuito del habla interna, donde se activan la percepción auditiva, el área de Wernicke (reconocimiento fonológico), el área de Broca (producción fonológica) y la producción articulatoria. A continuación, la Figura 5 representa el circuito del habla interna.

Figura 5:Circuito del habla interna

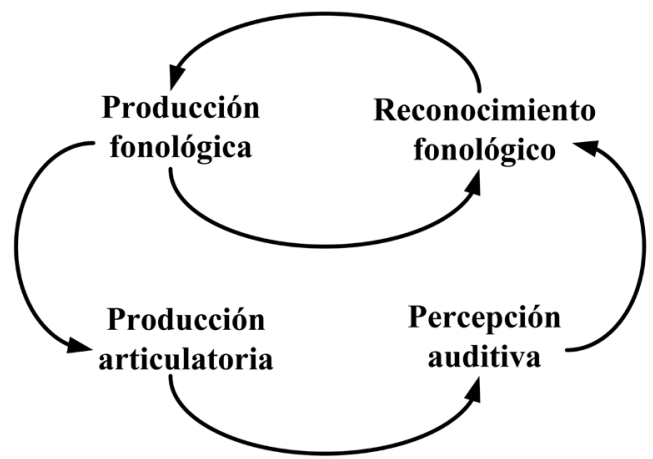

Fuente: tomado de Lamb (2011, p. 279).
La Figura 5 es corolario de buena parte del análisis que se ha efectuado hasta aquí. Antes, la Figura 2 representaba la información fundamental de la segunda sección de este trabajo, acerca de las rutas de la lectura. En ella se mostraba, entre otras cosas, que el estímulo visual generado por la letra escrita era tratado primero por la percepción visual y luego por el reconocimiento ortográfico. Se planteó en el título sobre conciencia fonológica que el aprendizaje de los grafemas requiere previamente del reconocimiento de los fonemas. Por lo tanto, el reconocimiento ortográfico tiene que estar conectado con el sistema de reconocimiento fonológico. A continuación, la Figura 6 ilustra estas conexiones. Obsérvese, además, que tiene que haber una conexión bidireccional entre el reconocimiento fonológico y el reconocimiento ortográfico. En primer lugar, y como ya se ha dicho, el reconocimiento de los grafemas es posible gracias al reconocimiento de los fonemas. De forma complementaria, y especialmente en el caso de los lectores expertos, el reconocimiento de los grafemas termina incidiendo en el reconocimiento de los fonemas (Ehri y Wilce, 1980; Castles y Colheart, 2004).

\section{Figura 6: Ruta de la lectura y su conexión con el circuito del habla interna}

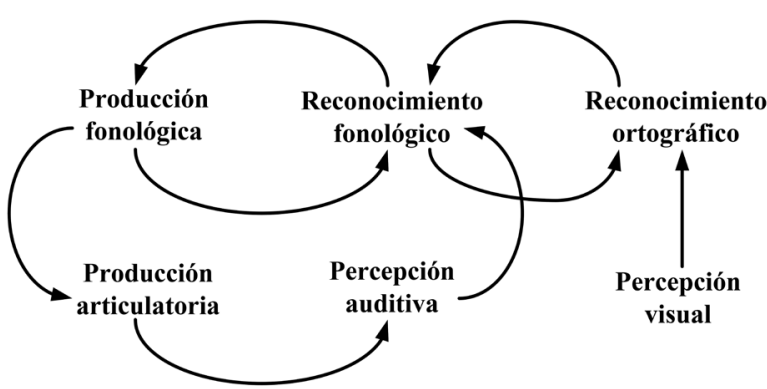

Fuente: elaboración propia.

Así las cosas, la lectura se constituye como un sistema perceptivo conectado al circuito del habla interna, que incluye tanto los dos sistemas fonológicos como la producción articulatoria y la producción auditiva. Por su parte, la escritura constituye un sistema de producción que tiene que estar conectado 
con el sistema de la lectura y con los sistemas del circuito del habla interna, vinculados a la lectura. En efecto, la Figura 6 (que no da cuenta de la escritura en tanto sistema productivo) muestra que, en la interpretación de la letra escrita, la percepción visual se conecta con el reconocimiento ortográfico y que este, a su vez, se conecta de forma bidireccional con el reconocimiento fonológico.

A continuación, la Figura 7 incorpora la representación de la producción ortográfica: este sistema tiene que estar conectado al área de la corteza motora que rige los movimientos de la mano con la que se escribe.

\section{Figura 7: Circuito de la lectoescritura interna}

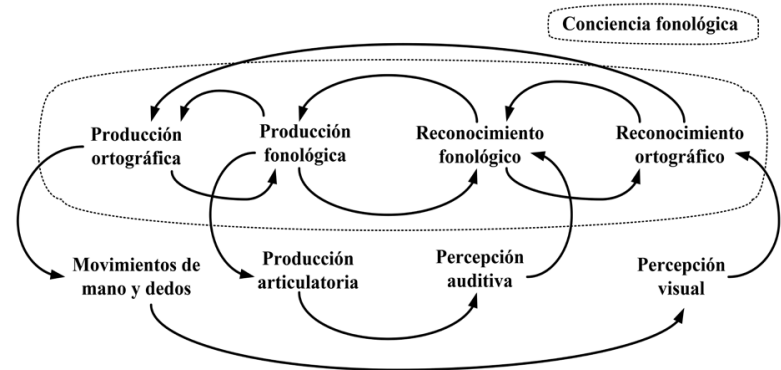

Fuente: elaboración propia.

Sobre la base de las consideraciones hasta aquí efectuadas, la Figura 7 sirve para dar cuenta de varias cuestiones. En primer lugar, la producción ortográfica se conecta bidireccionalmente con la producción fonológica. En efecto, cuando una persona escribe tiene que reconocer el fonema que se identifica con el grafema en cuestión. Y así como la lectura incide en el reconocimiento fonológico, es de esperar que la producción ortográfica incida en la producción fonológica.

Un ejemplo en verdad extraño, casi patético, pero contundente de cómo la producción ortográfica puede llegar a incidir en la producción fonológica se corresponde con un caso de la enseñanza de la ortografía en Argentina. En una época llegó a enseñarse, en contra de la intuición más básica del reconocimiento fonológico y de la producción fonológica reales, que la "v" (ve corta) era labioden- tal, mientras que la "b" (be larga) era bilabial. Así, quienes escribíamos dictados teníamos que prestar atención a las diferencias articulatorias que de forma ciertamente impostada iban surgiendo en los enunciados de la maestra. La postulación de esta diferencia es un error porque los grafemas "b" $y$ " $v$ " se corresponden ambos con el fonema /b/y no hay en el castellano un fonema cuyos rasgos articulatorios sean labiodental, fricativo, sonoro.

Un ejemplo más convencional y útil de la incidencia de la producción ortográfica en la producción fonológica es el conocido hecho de que los hablantes manifiestan la tendencia a marcar su dicción y a pronunciar los sonidos finales de palabras como $1 i$ bertad o andaluz cuando son conscientes de que su lectura en voz alta está siendo escuchada por otros.

En la Figura 7 también puede advertirse que el reconocimiento ortográfico monitorea la producción ortográfica. Esta hipótesis resulta muy plausible sobre la base del supuesto confirmado según el cual la percepción monitorea la producción. Además, la Figura 7 es consistente con la idea de que la producción participa en los procesos perceptivos. Por lo tanto, cabe esperar que el área motora que rige el movimiento de la mano y los dedos active el área de la percepción visual. Evoquemos mentalmente la escritura de la letra "A", tal vez con los ojos cerrados, y experimentemos cómo en efecto se activa la percepción visual de esa letra.

En conclusión, alrededor del circuito del habla interna se termina configurando el circuito de la lectoescritura interna. La Figura 7 no solo representa los subsistemas que participan en ese circuito, sino que también permite entender el ámbito de la conciencia fonológica. En efecto, la línea entrecortada de la Figura 7 abarca los cuatro subsistemas de la parte superior: 1) producción ortográfica, 2) producción fonológica, 3) reconocimiento fonológico, 4) reconocimiento ortográfico. Es así que la conciencia fonológica puede concebirse como la serie de interconexiones entre estos cuatro subsistemas, con particular énfasis entre los dos últimos para el caso de la lectura. 
Así las cosas, en el nivel inicial se podrá estimular el surgimiento o el desarrollo de la conciencia fonológica para consolidar luego el aprendizaje de la lectoescritura y prevenir dificultades. Como se ha dicho, la evidencia neuropsicológica permite confirmar la hipótesis de que la conciencia fonológica resulta fundamental para el aprendizaje de la lectoescritura. Desde luego, aunque la conciencia fonológica es en verdad necesaria para aprender a leer, no habrán de descuidarse otros aspectos fundamentales como la fluidez, el vocabulario y la comprensión, todos los cuales participan en la formación de lectores expertos.

Dicho sea de paso, el análisis de la lectoescritura en tanto sistema neurocognitivo y el análisis de la importancia de la conciencia fonológica contribuyen también a entender la ineficacia y aun la vacuidad del conocido método global para la enseñanza de la lectura. Por ejemplo, según el método global, un ejercicio viable consiste en que el niño identifique la correspondencia entre una palabra escrita y un dibujo a partir de los contornos de las letras. Pero en verdad ejercicios como ese no tienen nada que ver con el modo en que nuestro cerebro reconoce la letra escrita. Estas consideraciones no dejan de ser pertinentes, porque el método global sigue impregnado en los libros de texto y en los materiales de instrucción de muchos países (Moats, 1999; 2000; Dehaene, 2009). No hay de hecho ninguna razón para llegar a creer que los contornos globales de las palabras desempeñan algún rol en la lectura. No reconocemos una palabra impresa a través de una comprensión holística de su contorno, sino porque nuestro cerebro la divide en letras o grafemas.

Sí es verdad que el área de la lectura, en la corteza occipito-temporal izquierda, procesa todas las letras de una palabra en paralelo. El interés en ese procesamiento veloz y paralelo es lo que probablemente explique por qué muchos docentes y psicólogos bien intencionados y aun respetados hayan defendido el método global. Pero hay un error en esa defensa, porque la inmediatez o la globalidad de la lectura es solo una ilusión generada por la rapidez con la que nuestros sistemas neurocognitivos llegan a manejar las secuencias.

En todo caso, cuando una persona llega a ser una lectora experta ya no lee "letra por letra" simplemente porque la percepción global (o "macropercepción") favorece la "micropercepción" de la secuencia escrita. Sin embargo, cuando está aprendiendo, el niño de 5-6 años tiene que empezar a reconocer letra por letra para luego estar en condiciones de reconocer la palabra completa, por así decirlo. En efecto, la enseñanza minuciosa y explícita de las correspondencias entre grafemas y fonemas les permite a los niños llegar a tener un dominio genuino del sistema de escritura alfabética. Y cuanto más rápido automatice la ruta letra-sonido, mejor posicionado estará un niño para poder llegar a concentrarse en el significado de lo que lee.

Una amplia brecha separa los conocimientos obtenidos gracias a las neurociencias de las prácticas en el aula. En términos prácticos, el fascinante problema de cómo enseñar a leer seguirá siendo más asunto de los maestros que de los neurocientíficos. De todas formas, los avances de las neurociencias no pueden deslindarse de los grandes debates pedagógicos. En función de lo aquí expuesto, puede sugerirse entonces que, si bien cada niño es único e irrepetible, cuando se trata de aprender a leer todos tienen estructuralmente el mismo cerebro, que impone las mismas restricciones y las mismas secuencias de aprendizaje.

Los hallazgos de las neurociencias son desde luego muy valiosos. Sin embargo, dicen poco o nada acerca de cómo dar clase. En un mundo cada vez más organizado alrededor de la inquietante ubicuidad de las nuevas tecnologías, no es impertinente (ni innecesario, ni obvio) justificar por qué los docentes del nivel inicial deberían seguir reforzando el desarrollo de la conciencia fonológica por medio de adivinanzas, poemas y canciones para que los niños puedan aprender a leer. Aunque se las pueda catalogar de convencio- 
nales, tradicionales y hasta de anticuadas, las buenas prácticas seguirán siendo buenas y no deberían abandonarse tan solo porque no son innovadoras.

Las maestras jardineras han venido estimulando la conciencia fonológica desde siempre, en la gran mayoría de los casos sin haber estudiado a fondo cómo se organiza y cómo funciona el cerebro lector. Gracias al estímulo de la conciencia fonológica, entonces, han ayudado enormemente a los niños a que puedan aprender a leer. Ahora bien, si las maestras jardineras estudian cómo es que el cerebro aprende a leer, podrán seguir llevando adelante la misma tarea de siempre, pero mejor.

\section{Referencias}

Adams, M. J. (1990). Beginning to read: Thinking and learning about print. Cambridge: MIT Press.

Azcoaga, J. E., Derman, B. e Iglesias, P. (1997). Alteraciones del aprendizaje escolar. Diagnóstico, fisiopatología y tratamiento. Buenos Aires: Paidós.

Benasich, A. A. y Tallal, P. (2002). Infant discrimination of rapid auditory cues predicts later language impairment. Behavioral and Brain Research, 136, 31-49. DOI: https://doi.org/10.1016/S0166-4328(02)ooo98-o

Benzon, W. (2016). Some thoughts on lexemes, the dome, and inner speech. Language under Discussion, 4,73-77. Recuperado de http://ludjournal.org/index.php?journal=LUD\&page=article\&op=view\&path\%5B\%5D=57

Betherton, L. y Holmes, V. M. (2003) The relationship between auditory temporal processing, phonemic awareness, and reading disability. Journal of Experimental Child Psychology, 84, 218-243. DOI: https://doi. org/10.1016/So022-0965(03)00023-7

Bird, J., Bishop, D. V., Freeman, N. (1995). Phonological awareness and literacy development in children with expressive phonological impairments. Journal of Speech and Hearing Research, 38(2), 446-462. DOI: https:// doi.org/10.1044/jshr.3802.446

Bizama, M., Arancibia, B. y Sáez, K. (2011). Evaluación de la conciencia fonológica en párvulos de nivel transición 2 y escolares de primer año básico, pertenecientes a escuelas de sectores vulnerables de la provincia de Concepción, Chile. Onomázein, 23(1), 81-103. Recuperado de https://www.redaly c.org/articulo. oa?id=134518490004

Bizama, M., Arancibia, B. y Sáez, K. (2013). Intervención psicopedagógica temprana en conciencia fonológica como proceso metalingüístico a la base de la lectura en niños de 5 a 6 años socialmente vulnerables. Estudios Pedagógicos, 39(2), 25-39. DOI: https://doi.org/10.4067/So718-07052013000200002

Blachman, B. A. (1989). Phonological awareness and word recognition: Assesment and intervention. En A. G. Kamhi y H. W. Catts (eds.), Reading disabilities: a developmental language perspective (pp. 133- 158). Boston: College-Hill Press.

Boder, E. (1973). Developmental dyslexia: A Diagnosis approach based on three atypical reading-spelling patterns. Developmental Medical and Child Neurology, 15(5), 663. DOI: https://doi.org/10.1111/j.1469-8749.1973.tbo5180.x 
Borges, J. L. (1941). Pierre Menard, autor del Quijote. En Obras Completas, 1923-1974 (pp. 440-450), Buenos Aires: Emecé, 1974.

Bravo Valdivieso, L. (2002). La conciencia fonológica como una zona de desarrollo próximo para el aprendizaje inicial de la lectura. Estudios Pedagógicos, 28, 165-177. DOI: https://doi.org/10.4067/So71807052002000100010

Carrillo Gallego, M. y Alegría Isoca, J. (2009). Exploración de las habilidades fonológicas en escolares disléxicos: teoría y práctica. Revista de Logopedia, Foniatría y Audiología, 29(2), 115-130. DOI: https://doi.org/10.1016/ So214-4603(09)70149-4

Castles, A., y Coltheart, M. (2004). Is there a causal link from phonological awareness to success in learning to read? Cognition 91(1), 77-111. DOI: https://doi.org/10.1016/S0010-0277(03)00164-1

Catts, H.W. (1993). The relationship between speech language impairments and reading disabilities. Journal of Speech and Hearing Research, 36, 948-958. DOI: https://doi.org/10.1044/jshr.3605.948

Catts, H. W. (1996). Defining dyslexia as a developmental language disorder: An expanded view. Topics in Language Disorders, 16, 14-29. Recuperado de https://journals.lww.com/topicsinlanguagedisorders/Abstract/1996/0200o/Defining_dyslexia_as_a_developmental_language.4.aspx

Cervera-Mérida, J. F. e Ygual-Fernández, A. (2003). Intervención logopédica en los trastornos fonológicos desde el paradigma psicolingüístico del procesamiento del habla. Revista de Neurología, 36(1), 39-53. Recuperado de http://www.sld.cu/galerias/pdf/sitios/rehabilitacion-logo/intervencion_logopedica_en_los_trastornos_fonologicos.pdf

Cheung, H. y Chen, H. C. (2004). Early orthographic experience modifies both phonological awareness and on-line speech processing. Language and Cognitive Processes, 19, 1-28. DOI: https://doi. org/10.1080/01690960344000071

Coloma Tirapegui, C., Cárdenas Gajardo, L. y De Barbieri, Z. (2005). Conciencia fonológica y lengua escrita en niños con trastorno específico del lenguaje expresivo. Revista Cefac, 7(4), 419-25. Recuperado de https:// www.redaly c.org/articulo.oa?id=169320507004

De Martino, S., Espesser, R., Rey, V.y Habib, M. (2001). The "temporal processing deficit" hypothesis in dyslexia: new experimental evidence. Brain and Cognition, 46(1-2), 104-108. DOI: https://doi.org/10.1016/So2782626(01)80044-0

Dehaene, S. (2009). Reading in the brain. The science and evolution of a human invention. Nueva York: Viking.

Ehri, L., y Wilce, L. (1980). The influence of orthography on readers' conceptualization of the phonemic structure of words. Applied Psycholinguistics, 1, 371-385. DOI: https://doi.org/10.1017/S0142716400009802

Fazio, B. B. (1997a). Memory for rote linguistic routines and sensitivity to rhyme: a comparison of low-income children with and without specific language impairment. Applied Psycholinguistics, 18, 347-374. DOI: https://doi.org/10.1017/S0142716400010511 
Fazio, B. B. (1997b). Learning a new poem: Memory for connected speech and phonological awareness in lowincome children with and without specific language impairment. Journal of Speech, Language and Hearing Research, 40, 1285-1297 DOI: https://doi.org/10.1044/jslhr.4006.1285

Ferraro, G., Caci, B., D’Amico, A., Di Blasi, M. (2007). Internet addiction disorder: an Italian study. Cyber Psychology and Behavior, 10(2), 170-175. DOI: https://doi.org/10.1089/cpb.2006.9972

Frith, U. (1985). Beneath the surface of developmental dyslexia. En K. E. Patterson, J. C. Marshall y M. Coltheart, (eds.), Surface dyslexia: cognitive and neuropsychological studies of phonological reading (pp. 301-330). Hillsdale, NJ: Erlbaum.

Galaburda, A. y Cestnick, L. (2003). Dislexia del desarrollo. Revista de Neurología, 36(1), 5-39. DOI: https://doi. org/10.33588/rn.36S1.2003068

Gallardo, B.y Sanmartín, J. (2005). Afasia fluente. Materiales para su estudio. Valencia: Guada.

Gil, J. M. (2017). Sobre el papel del lenguaje en el fracaso escolar. Didáctica. Lengua y Literatura, 29, 121-137. DOI: https://doi.org/10.5209/DIDA.57133

Gómez, L., Duarte, A. M., Merchán, V., Aguirre, D. y Pineda, D. (2007). Conciencia fonológica y comportamiento verbal en niños con dificultades de aprendizaje. Universitas Psychologica, 6(3), 571-580. Recuperado de: http://pepsic.bvsalud.org/scielo.php?script=sci_arttext\&pid=S1657-92672007000300009

Goodman, K. (1982). Language and literacy: Selected writings of Keneth Goodman. Londres: Routledge.

Goodman, K.y Goodman, Y. (1979). Learning to read is natural. En L. Resnick y P. Weaver (eds.), Theory and practice of early reading (pp.137-154). Hillsdale, NJ: Lawrence Erlbaum.

Goswami, U. (1986). Children's use of analogy in learning to read: A developmental study. Journal of Experimental Child Psychology, 42, 73-83. DOl: https://doi.org/10.1016/0022-0965(86)90016-o

Goswami, U., Gombert, J. E., y De Barrera, L. F. (1998). Children's orthographic representations and linguistic transparency: nonsense word reading in English, French, and Spanish. Applied Psycholinguistics, 19, 19-52. DOI: http://dx.doi.org/10.1017/S0142716400010560

Grigorenko, E. et al. (1997). Susceptibility loci for distinct components of developmental dyslexia on chromosomes 6 and 15. American Journal of Human Genetics, 60(1), 27-30. Recuperado de: https://www.ncbi.nlm. nih.gov/pmc/articles/PMC1712535/

Herrera, L. y Defior, S. (2005). Una aproximación al procesamiento fonológico de los niños prelectores: conciencia fonológica, memoria verbal a corto plazo y denominación. Psykhe, 14(2), 81-95. DOI: https://doi. org/10.4067/So718-22282005000200007

Hickock, G. (2014). The myth of mirror neurons: The real neuroscience of communication and cognition. Nueva York:W.W. Norton. 
Horwitz, B. Rumsey J. M. y Donohue, B. C. (1998). Functional connectivity of the angular gyrus in normal reading and dyslexia. National Academy Science USA, 95(15), 8939-8944. DOI: https://doi.org/10.1073/ pnas. 95.15 .8939

Hudson, R. (2016). Comments on 'Linguistic structure: A plausible theory'. Language Under Discussion, 4(1), 3843. Recuperado de: http://www.ludjournal.org/index.php?journal=LUD\&page=article\&op=view\&path\% $5 B \% 5 D=52$

James, K. H. y Engelhardt, L. (2012). The effects of handwriting experience on functional brain development in pre-literate children. Trends in Neuroscience and Education, 1(1), 32-42. DOI: https://doi.org/10.1016/j. tine.2012.08.001

Jiménez González, J. E. (coord.) (2012). Dislexia en español: prevalencia e indicadores cognitivos, culturales, familiares y biológicos. Madrid: Pirámide.

Johnson, D. J. y Myklebust, H. R. (1967). Learning disabilities: educational principles and practices. Nueva York: Grune \& Stratton.

Johnson, N. F. (2009). The multiplicities of Internet addiction: the misrecognition of leisure and learning. Surrey: Ashgate.

Kaminsky, M., Eviatar, Z. y Norman, J. (2002). The timing deficit hypothesis of dyslexia and its implications for Hebrew reading. Brain and Cognition, 48(3), 394-398. Recuperado de: https://www.ncbi.nlm.nih.gov/pubmed/12030475

Kinsbourne, M. y Warrington, E. K. (1962). A disorder of simultaneous form perception. Brain, 85, 461- 486. DOI: https://doi.org/10.1093/brain/85.3.461

Lamb, S. M. (2004). Language and reality. Londres: Continuum.

Lamb, S. M. (2011). Senderos del cerebro. La base neurocognitiva del lenguaje. Mar del Plata: Universidad de Mar del Plata.

Lamb, S. M. (2016a). Linguistic structure: A plausible theory. Language under Discussion, 4(1), 1-37. Recuperado de: http://www.ludjournal.org/index.php?journal=LUD\&page=article\&op=view\&path[]=30

Lamb, S. M. (2016b). Reply to comments. Language under Discussion, 4(1), 78-82. Recuperado de: http://www. ludjournal.org/index.php?journal=LUD\&page=article\&op=view\&path\%5B\%5D=60

Leybaert, J. (1998). Phonological representations in deaf children: the importance of early linguistic experience. Scandinavian Journal of Psychology, 39, 169-173. DOI: https://doi.org/10.1111/1467-9450.393074

Li, S.-M. y Chung, T.-M. (2006). Internet function and Internet addictive behavior. Computers in Human Behavior, 22, 1067-1071. DOI: https://doi.org/10.1016/j.chb.2004.03.030 
Liberman, I. (1988). ¿Deben las llamadas preferencias de modalidad determinar la naturaleza de la instrucción de los niños con dificultades para la lectura? En F. Duffy y N. Geschwind, Dislexia aspectos psicológicos y neurológicos. Barcelona: Labor.

Liberman, I., Shankweiler, D., Fischer, R. y Carter, B. (1974). Explicit syllable and phoneme segmentation in the young children. Journal of Experimental Child Psychology, 18, 201-212. DOI: https://doi.org/10.1016/00220965(74)90101-5

Lundberg, I., Frost, J. y Petersen, O. (1988). Effects of an extensive program for stimulating phonological awareness in preschool children. Reading Research Quarterly, 23(3), 263-284. DOI: https://doi.org/10.1016/o9594752(95)00004-M

Majumdar, A. y Sowa, J. (2014). Quantum cognition. Web Intelligence and Intelligent Agent Technologies, 2, 1-4. DOI: https://doi.org/10.1109/WI-IAT.2014.9

Mann, V.A. (1986). Phonological awareness: the role of reading experience. Cognition, 24(1-2), 65-92. DOI: https:// doi.org/10.1016/0010-0277(86)90005-3

Márquez, J. y De la Osa Fuentes, P. (2003). Evaluación de la conciencia fonológica en el inicio lector. Anuario de Psicología, 34(3), 357-370. Recuperado de https://dialnet.unirioja.es/servlet/articulo?codigo=722840

Mattis, S, French, J. y Rapin, I. (1975). Dyslexia in children and young adults: three independents neuropsy chological syndromes. Developmental Medical and Child Neurology, 17, 150. DOI: https://doi. org/10.1111/j.1469-8749.1975.tbo3467.x

Mejía de Eslava, L.y Eslava Cobos, J. (2008). Conciencia fonológica y aprendizaje lector. Acta Neurológica Colombiana, 24, 55-63. Recuperado de https://www.acnweb.org/acta/2008_24_S2_55.pdf

Merzenich, M. et al. (1996). Temporal processing deficits of language-learning impaired children ameliorated by training. Science, 271, 77-81. DOI: https://doi.org/10.1126/science.271.5245.77

Miller, P. (1997). The effect of communication mode on the development of phonemic awareness in prelingually deaf students. Journal of Speech, Language and Hearing Research, 40, 1151-1163. DOI: https://doi. org/10.1044/jslhr.4005.1151

Moats, L. C. (1999). Teaching reading is rocket science. Washington, DC: American Federation of Teachers.

Moats, L. C. (2000). Speech to print: Language essentials for teachers. Baltimore: Paul H. Brookes.

Morais, J., Bertelson, P., Cary, L.y Alegria, J. (1986). Literacy training and speech segmentation. Cognition, 24, 45-64. DOI: https://doi.org/10.1016/0010-0277(86)90004-1

Morais, J., Cary, L., Alegria, J. y Bertelson, P. (1979). Does awareness of speech as a sequence of phones arise spontaneously? Cognition, 7, 323-331. DOI: https://doi.org/10.1016/0010-0277(79)90020-9 
Nielsen, D. C. y Luetke-Stahlman, B. (2002). Phonological awareness: one key to the reading proficiency of deaf children. American Annals of the Deaf, 147, 11-19. DOI: https://doi.org/10.1353/aad.2012.0242

Núñez Delgado, M. P.y Santamarina Sancho, M. (2014). Prerrequisitos para el proceso de aprendizaje de la lectura y la escritura: conciencia fonológica y destrezas orales de la lengua. Lengua y Habla, 18, 72-92. Recuperado de: https://dialnet.unirioja.es/servlet/articulo?codigo=4888939

Orton, S. T. (1937). Reading writing and speech problems in children: A presentation of certain types of disorders in the development of the language Faculty. Nueva York: Norton.

Osborne, T. (2016). Dependency networks: A discussion note about Lamb's theory of linguistic structure. Language under Discussion, 4(1), 44-50. Recuperado de http://www.ludjournal.org/index.php?journal=LUD\& page $=$ article\&op $=$ view $\&$ path $\% 5 B \% 5 D=53$

Paul, R., Murray, C., Clancy, K.y Andrews, D. (1997). Reading and metaphonological outcomes in late talkers. Journal of Speech, Language and Hearing Research, 40, 1037-1047. DOI: https://doi.org/10.1044/jslhr.4005.1037

Pinker, S. (1994). The language instinct. Nueva York: Morrow.

Pirozzolo, F. J. (1979). The neuropsychology ofdevelopmental reading disorders. Nueva York: Praeger.

Rastall, P. (2016). Is linguistic structure an illusion? Language under Discussion, 4(1), 51-72. Recuperado de: http:// www.ludjournal.org/index.php?journal=LUD\&page =article\&op=view\&path\%5B\%5D=54

Rayner, K., Foorman, B. R., Perfetti, C. A., Pesetsky, D., y Seidenberg, M. S. (2001). How psy chological science informs the teaching of reading. Psychological Science in the Public Interest, 2, 31-74. Recuperado de https://www. researchgate.net/publication/2590161_How_psy chological_science_informs_the_teaching_of_reading

Rayner, K.y Pollatsek, A. (1989). The psychology of reading. Englewood Cliffs: Prentice Hall.

Read, C., Zhang, Y. F., Nie, H. Y.y Ding, B. O. (1986). The ability to manipulate speech sounds depends on knowing alphabetic writing. Cognition, 24(1-2), 31-44. DOI: https://doi.org/10.1016/0010-0277(86)90003-X

Reigosa, V., Pérez-Abalo, M. C., Piñeiro, A. y López, I. (2002). Fiabilidad y validez de una batería de pruebas computarizadas (BTL) para la evaluación cognitiva de la lectura. Revista Latina de Pensamiento y Lenguaje y Neuropsicología Latina, 10, 229-248. Recuperado de https://scholar.google.com/citations?user=L798EPYA AAAJ\&hl=en

Rizzolatti, G. y Sinigaglia, C. (2015). Curious book on mirror neurons and their myth, The American Journal of Psychology, 128(4), 527-53. DOI: https://doi.org/10.5406/amerjpsy c.128.4.0527

Sastre-Gómez L., Celis-Leal N., Roa de la Torre J. D.y Luengas-Monroy C. F. (2017). La conciencia fonológica en contextos educativos y terapéuticos: efectos sobre el aprendizaje de la lectura. Educación y Educadores, 2O(2), 175-19o. DOI: https://doi.org/10.5294/edu.2017.20.2.1 
Shaywitz, S. E., Shaywitz, B. A., Pugh, K. R. y Fullbright, R. K. (1998). Functional disruption in the organization of the brain for reading in dyslexia. National Academy of Sciences USA, 95(5), 2636-2641. DOI: https://doi. org/10.1073/pnas.95.5.2636

Solsona, J., Navarro, J. I. y Aguilar, M. (2005). Conocimiento lógico matemático y conciencia fonológica en educación infantil. Revista de Educación, 341, 781-801. Recuperado de https://www.researchgate.net/publication/28132782_Conocimiento_logico-matematico_y_conciencia_fonologica_en_Educacion_Infantil

Sowa, J. (2016). The virtual reality of the mind. Procedia Computer Science, 88, 139-144. DOI: https://doi. org/10.1016/j.procs.2016.07.417

Spitzer, M. (2014). Information technology in education: risks and side effects. Trends in Neuroscience and Education, 3(3-4), 81-85. DOI: DOI: https://doi.org/10.1016/j.tine.2014.09.002

Sterne, A. y Goswami, U. (2000). Phonological awareness of syllables, rhymes, and phonemes in deaf children. Journal of Child Psychology and Psychiatry, and allied Disciplines, 41, 609-625. DOI: https://doi. org/10.1111/1469-7610.00648

Tallal, P. (1980). Auditory temporal perception, phonics and reading disabilities in children. Brain and Language, 9, 182. DOI: https://doi.org/10.1016/0093-934X(80)90139-X

Tallal, P. et al.(1996). Language comprehension in language-learning impaired children improved with acoustically modified speech. Science, 271, 27-28.

Tallal, P., Stark R. y Mellits, E. (1985). Identification of language impairment children on the basis of rapid perception and production skills. Brain and Language, 25(2), 314-322. DOI: https://doi.org/10.1016/0093$934 \times(85) 90087-2$

Torgesen, J., Morgan, S.y Davis, C. (1992). Effects of two types of phonological awareness training on word learning in kindergarten children. Journal of Educational Psychology, 84, 364-370. DOI: http://doi.org/10.1037/00220663.84.3.364

Treiman, R. y Zukowski, A. (1991). Levels of phonological awareness. En S. Brady, D. Shankweiler (eds.), Phonological processes in literacy (pp. 67-84). Hillsdale: Lawrence Erlbaum.

Trías, D., Cuadro, A. y Costa, D. (2009). Desarrollo de la conciencia fonémica: evaluación de un programa de intervención. Ciencias Psicológicas, 3(2), 177-184. Recuperado de https://revistas.ucu.edu.uy/index.php/cienciaspsicologicas/article/view/149/

Vargas, A. y Villamil, W. (2007). El papel de la conciencia fonológica como habilidad subyacente al alfabetismo temprano y su relación en la comprensión de la lectura y la producción escrita de textos. Pensamiento Psicológico, 3(9), 163-174. Recuperado de https://www.redaly c.org/pdf/801/80103912.pdf

Waber, D. P. et al.(2002). Processing of rapid auditory stimuli in school-age children referred for evaluation learning disorders. Child Developmental, 72, 37-49. DOl: https://doi.org/10.1111/1467-8624.00264 
Wu, C.-S.y Cheng, F.-F. (2007). Internet cafe addiction of Taiwanese adolescents. Cyber Psychology and Behavior, 10(2), 220-225. DOI: https://doi.org/10.1089/cpb.2006.9965

Yopp, H. K. (1988). The validity and reliability of phonemic awareness tests. Reading Research Quarterly, 23, 159177. DOI: http://doi.org/10.2307/747800

Young, K. S. (2007). Cognitive behavior therapy with internet addicts: treatment outcomes and implications. CyberPsychology and Behavior, 10(5), 671-679. DOI: https://doi.org/10.1089/cpb.2007.9971

Yubero, S., Larrañaga, E., Navarro, R., y Elche, M. (2018). Padres, hijos e Internet. Socialización familiar de la Red. Una relación compleja. Universitas Psychologica, 17 (2), 1-13. DOI: https://doi.org/10.11144/Javeriana.upsy17-2.phis 